\title{
Associação entre a ocorrência de dor e sobrecarga em cuidadores principais e o nível de independência de idosos nas atividades de vida diária: estudo transversal*
}

\section{Association between the occurrence of pain and caregivers overload on main and the elderly independence level in daily life activities: cross sectional study}

Cristine Cogo Lopes ${ }^{1}$, Gabriela Almeida de Oliveira' ${ }^{1}$ Felipe de Souza Stigger ${ }^{2}$,

Adriana Torres de Lemos²

'Universidade Federal de Ciências da Saúde de Porto Alegre - Porto Alegre (RS), Brasil.

${ }^{2}$ Departamento de Fisioterapia, Universidade Federal de Ciências da Saúde de Porto Alegre - Porto Alegre (RS), Brasil.

Como citar: Lopes CC, Oliveira GA, Stigger FS, Lemos AT. Associação entre a ocorrência de dor e sobrecarga em cuidadores principais e o nível de independência de idosos nas atividades de vida diária: estudo transversal. Cad Saúde Colet, 2020;28(1):98-106. https://doi.org/10.1590/1414-462X202028010184

\section{Resumo}

Introdução: Cuidar do idoso costuma ser uma tarefa árdua, e as demandas podem extrapolar a capacidade do cuidador, causando sobrecarga e dor. Objetivo: Investigar a ocorrência de sobrecarga e dor em cuidadores de idosos, bem como verificar a relação dessas variáveis com o nível de independência do idoso. Método: Amostra por conveniência que, a partir de Unidades de Saúde de uma região distrital de Porto Alegre, identificou potenciais idosos e seus cuidadores principais. Estes, em visitas domiciliares, responderam questões referentes ao perfil socioeconômico, à ocorrência de dor (questionário nórdico e escala visual analógica de dor), à sobrecarga do cuidador (Inventário de Sobrecarga do Cuidador) e ao nível de independência do idoso (índice de Katz). Foi realizada correlação de Spearman. Resultados: Participaram 14 idosos e 14 cuidadores com idade média de $82,1( \pm 6,9)$ e 65,9( $\pm 11,3)$ anos, respectivamente. Dos idosos, $28,6 \%$ foram classificados independentes, $50 \%$ dependência moderada e $21,4 \%$ muito dependentes. Houve correlação forte e inversa entre o nível de independência do idoso e a sobrecarga do cuidador $(\rho=-0,619 ; p=0,018)$ e com os domínios, separadamente, sobrecarga tempo dependente $(\rho=-0,866 ; p=0,000)$ e sobrecarga à vida pessoal $(\rho=-0,708 ; p=0,005)$. Conclusão: Quanto mais independente o idoso, menor foi a sobrecarga atribuída ao seu cuidador. Não houve associação entre dor e sobrecarga do cuidador.

Descritores: envelhecimento; atividades cotidianas; cuidadores; promoção da saúde.

\begin{abstract}
Background: Caring for an elderly is often an arduous task, and the care demands go beyond the caregiver's capacity may cause overload and pain. Objective: The aim of this study was to investigate the association between independence level of the elderly in daily life activities and the occurrence of pain and overload your primary caregiver. Method: The study included 14 elderly residents in adscript territory of health units of the North Zone-Axis Baltazar area Baltazar North axis of the city Porto Alegre and their primary caregivers, in home visits, answered questions regarding the socioeconomic profile, the occurrence of pain (Nordic questionnaire and visual analog pain scale), the caregiver burden (Inventory
\end{abstract}

\footnotetext{
* Visitas domiciliares aos participantes, estes pertencentes ao Distrito Docente Assistencial da UFCSPA.
}

Trabalho realizado na Universidade Federal de Ciências da Saúde de Porto Alegre (UFCSPA) - Porto Alegre (RS), Brasil Correspondência: Adriana Torres de Lemos. E-mail: adrianatl@ufcspa.edu.br

Fonte de financiamento: nenhuma.

Conflito de interesses: nada a declarar.

Recebido em: Mar. 26, 2018. Aprovado em: Maio 14, 2019

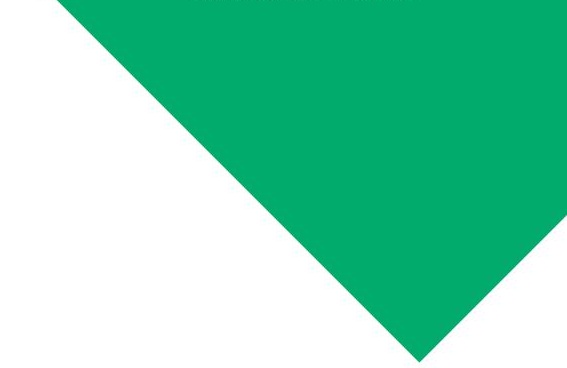


caregiver overload) and the level of independence of the elderly (Katz index). Results: The average age of the elderly is 82.1 years $( \pm 6.9)$ and caregivers, 65.9 years $( \pm 11.3)$. Of the 14 elderly, $28.6 \%$ were classified as independent, $50 \%$ as having moderate dependence and $21.4 \%$ as very dependent. There was strong inverse correlation between the old level of independence and caregiver burden $(\rho=-0.619$; $p=0.018)$. When evaluating areas of the inventory separately overload time dependent $(\rho=-0.866$; $p=0.000)$ and over the personal life $(p=-0.708 ; p=0.005)$ correlated to the level of independence of the elderly. Conclusion: The study showed that the more independent for the elderly, the lower the burden attributed to the caregiver.

Keywords: aging; activities of daily living; caregivers; health promotion.

\section{INTRODUÇÃO}

O intenso ritmo de envelhecimento da população brasileira tem suscitado diversos debates, especialmente sobre o aumento das demandas dos serviços de saúde e o desafio de buscar alternativas de cuidado ao idoso' ${ }^{1}$.Tais debates não emergem exclusivamente devido à crescente parcela da população idosa, mas sim pelo fato dessa população estar mais exposta a doenças degenerativas com potencialidade para afetar as habilidades físicas e mentais necessárias para realização de atividades básicas e instrumentais da vida diária, tornando-os dependentes ${ }^{2}$. Dessa forma, à medida que ocorre o comprometimento das capacidades funcionais, o idoso necessita de assistência de outra pessoa para o desempenho de suas atividades e tarefas cotidianas, o cuidador ${ }^{3}$.

Podemos definir o cuidador, segundo a Classificação Brasileira de Ocupações (CBO), como a pessoa responsável pelo ato de cuidar, de acordo com objetivos estabelecidos por instituições especializadas ou pelos responsáveis diretos, assim como zelar pelo bem-estar, saúde, alimentação, higiene pessoal, educação, cultura, recreação e lazer do indivíduo assistido 4 . Quando os cuidadores prestam serviços sob remuneração, são considerados cuidadores formais; já são considerados informais quando qualquer tipo de cuidado é prestado por um membro da família ou da comunidade de forma voluntária ${ }^{5}$. Ainda, o cuidador pode ser considerado como cuidador principal quando exerce a total ou a maior responsabilidade pelos cuidados prestados ao idoso no domicílio. Já os cuidadores secundários frequentemente são os familiares, voluntários ou profissionais que prestam atividades de forma complementar ${ }^{6}$.

A experiência de assumir a responsabilidade por idosos dependentes tem sido colocada pelos cuidadores familiares como uma tarefa exaustiva e estressante, tendo em vista o envolvimento afetivo e a transformação de uma relação de dependência, em que o cuidador passa a ter restrições em relação à sua própria vida ${ }^{7}$.

Ao assumir a responsabilidade de cuidar de uma pessoa idosa, cuidadores geralmente o fazem de maneira ininterrupta e sem descanso. Normalmente o cuidador trabalha muitas horas seguidas, principalmente com atividades relacionadas aos cuidados pessoais de higiene, alimentação, transferências, assim como outras situações que ocasionam situações estressantes e sobrecarga ${ }^{8}$.

Ainda, o atributo de cuidado a um idoso dependente costuma ser delegado a uma única pessoa, o que impõe inúmeras exigências ao cuidador, podendo potencializar a sobrecarga, o que muitas vezes leva o cuidador ao adoecimento físico e mental ${ }^{6,9}$.

A sobrecarga de cuidar de um idoso é bem documentada na literatura existente $\mathrm{e}^{10-12}$ A literatura refere que os cuidadores sentem-se sobrecarregados devido às alterações sociais, profissionais e pessoais que o cuidado impõe nas suas vidas. Além disso, existe uma escassa e inadequada formação e informação em relação à patologia de base e ao cuidado de idosos dependentes ${ }^{13}$, sendo de suma importância que sejam fornecidas informações sobre saúde e manejo do idoso, e instruções sobre autocuidado, importante para evitar um ciclo de negligência, em que esses cuidadores estariam futuramente suscetíveis à dependência, necessitando de um novo cuidador ${ }^{4,14}$. Diante disso, o presente estudo tem como objetivo investigar a ocorrência de sobrecarga e dor em cuidadores de idosos, bem como verificar a relação dessas variáveis com o nível de independência do idoso. 


\section{MÉTODO}

Trata-se de um estudo observacional transversal com cuidadores principais de idosos residentes no território adscrito de unidades de saúde de uma região distrital da cidade de Porto Alegre. As unidades de saúde, eleitas por critério de conveniência (aceite em participar pela Coordenação da Unidade e facilidade de acesso às residências sem a necessidade de acompanhamento de agentes de saúde), indicaram potenciais participantes para o estudo a partir de levantamento realizado com auxílio da equipe de saúde, respeitando os critérios de inclusão (autodenominar-se cuidador principal de uma pessoa com idade igual ou superior a 60 anos; idosos residentes no território adscrito das unidades de saúde selecionadas; prestar cuidados ao idoso em turno diurno) e de exclusão (ser cuidador formal, ou seja, prestar serviços sob remuneração; cuidador com idade inferior a 18 anos; prestar assistência a mais de um idoso).

Através de contato telefônico, idosos e seus respectivos cuidadores principais foram convidados a participar do estudo, e, nos casos de negativa, não houve novo contato. Para os casos de aceite, era solicitado o endereço da residência e montado o roteiro de coleta dos dados conforme proximidade geográfica das moradias. Com o roteiro estabelecido, fez-se novo contato telefônico para agendamento da visita domiciliar, as quais ocorreram nos meses de maio e junho de 2016. O período de intervalo entre o contato telefônico e a visita foi de, no máximo, duas semanas.

Em visitas domiciliares, os participantes eram esclarecidos sobre o estudo e, então, assinavam o Termo de Consentimento Livre e Esclarecido (TCLE). Quando o idoso era incapaz de responder por si, o cuidador principal era quem o fazia. Após, prosseguia-se com entrevista para preenchimento dos questionários de caracterização sociodemográfica, avaliação de sobrecarga e dor do cuidador e avaliação do nível de independência do idoso nas Atividades de Vida Diária (AVDs). As doenças, autorreferidas, foram categorizadas pelos pesquisadores para fins de apresentação das frequências de ocorrência.

A sobrecarga do cuidador foi avaliada através do Inventário de Sobrecarga do Cuidador ${ }^{15}$, sendo analisado tanto seu escore total quanto seus cinco domínios separadamente (tempo dependente, vida pessoal, física, social e emocional), considerando maior sobrecarga quanto mais alta a pontuação. Para avaliar ocorrência e intensidade de dor no cuidador, foram utilizados dois instrumentos: Questionário Nórdico ${ }^{16,17}$ e Escala Visual Analógica (EVA) ${ }^{18}$. A partir do Questionário Nórdico, foi possível identificar em quais regiões corporais os cuidadores tinham dor e, pela EVA, qual a respectiva intensidade. Nos casos em que houve referência de dor no Questionário Nórdico em muitas regiões, foi considerada, para fins de análise, a média entre os três locais em que o sujeito havia apontado maior intensidade na EVA.

A avaliação do nível de independência do idoso foi através do Índice de Katz ${ }^{19}$, no qual, quanto maior a pontuação, maior a independência do idoso.

Para a caracterização da amostra, foi utilizada estatística descritiva através de média e desvio-padrão para as variáveis contínuas, e, para as variáveis categóricas, a frequência de ocorrência. A associação entre o nível de independência nas AVDs do idoso e a sobrecarga e a ocorrência e nível de dor no cuidador foi avaliada pela correlação de Spearman, devido ao tamanho amostral. A normalidade dos dados foi avaliada pelo Teste de Shapiro-Wilk. As análises foram realizadas utilizando o pacote estatístico SPSS 17.0, e foi considerado nível de significância de $5 \%$.

Este estudo teve aprovação de Comitês de Ética em Pesquisa sob os números 1.379.265 e 1.451.163.

\section{RESULTADOS}

Foram identificados 26 possíveis pares (idosos e seus respectivos cuidadores) para participarem do estudo. Destes, dois indivíduos que recebiam cuidados não atendiam aos critérios de inclusão de ter mais de 60 anos, sendo 24 pares elegíveis para o contato telefônico inicial. Após contato inicial, seis cuidadores negaram participação, dois idosos eram independentes sem necessidade de nenhum tipo de cuidado e, daqueles que aceitaram participar, dois idosos faleceram entre o contato inicial e o telefonema para marcação da visita. Assim, a amostra foi composta por 14 pares. A Figura 1 mostra o fluxograma de captação dos participantes. 


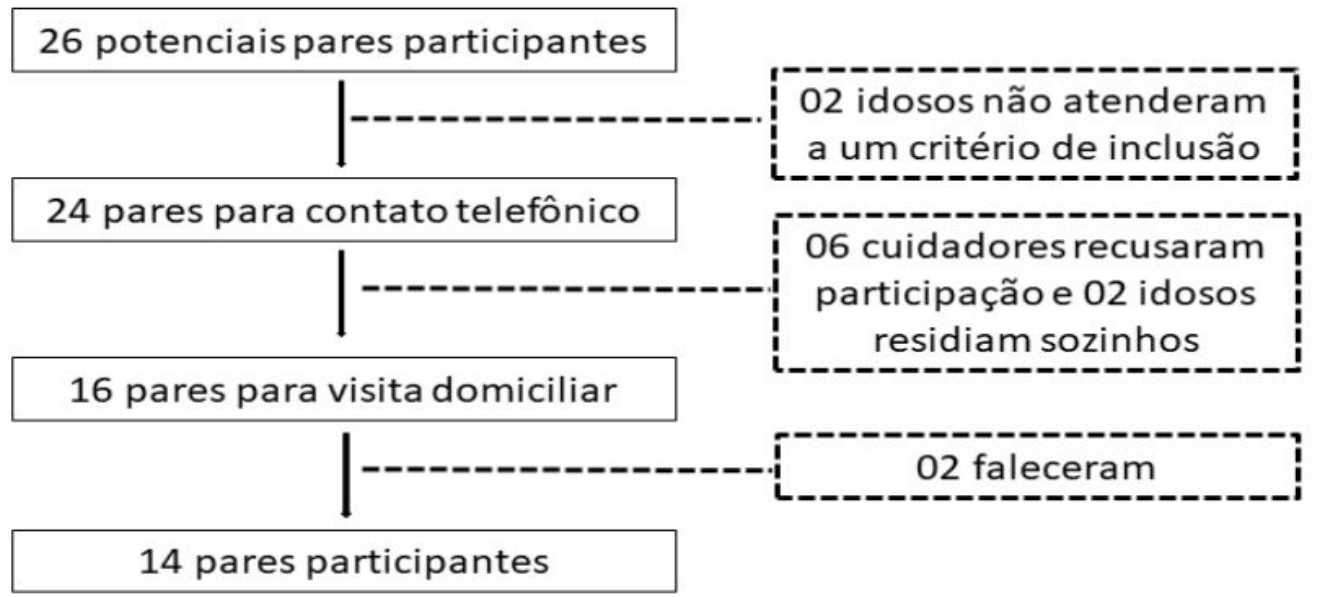

Figura 1. Fluxograma de captação dos participantes. Porto Alegre - RS, 2016

A média de idade dos idosos foi de 82,1 $( \pm 6,9)$ anos, com idade mínima de 64 anos e máxima de 95 anos. Os cuidadores tinham em média 65,9 ( $\pm 11,3)$ anos, sendo a idade mínima 44 e a máxima 83 anos. Quanto ao Índice de Massa Corporal (IMC), a média foi de $23,8( \pm 3,9) \mathrm{kg} / \mathrm{m}^{2}$ para os idosos e de $26,4( \pm 3,8) \mathrm{kg} / \mathrm{m}^{2}$ para os cuidadores. A Tabela 1 apresenta a caracterização da amostra em relação ao sexo, estado civil e escolaridade.

Tabela 1. Caracterização da amostra em relação ao sexo, estado civil e escolaridade. Porto Alegre - RS, 2016

\begin{tabular}{|c|c|c|c|}
\hline \multirow{2}{*}{ Variáveis } & & Idoso & Cuidador \\
\hline & & $N(\%)$ & $N(\%)$ \\
\hline \multirow{2}{*}{ Sexo } & Feminino & $8(57,1)$ & $10(71,4)$ \\
\hline & Masculino & $6(42,9)$ & $4(28,6)$ \\
\hline \multirow{4}{*}{ Estado Civil } & Solteiro & - & $4(28,6)$ \\
\hline & Casado & $7(50)$ & $7(50)$ \\
\hline & Viúvo & $5(35,7)$ & $3(21,4)$ \\
\hline & Divorciado & $2(14,3)$ & - \\
\hline \multirow{5}{*}{ Escolaridade } & Não Alfabetizado & $4(28,6)$ & - \\
\hline & $1^{\circ}$ grau incomp. & $9(64,3)$ & $7(50)$ \\
\hline & $1^{\circ}$ grau comp. & - & $1(7,1)$ \\
\hline & $2^{\circ}$ grau incomp. & - & $1(7,1)$ \\
\hline & $2^{\circ}$ grau comp. & $1(7,1)$ & $5(35,7)$ \\
\hline
\end{tabular}

Quanto ao perfil sociodemográfico dos idosos, 85,8\% são aposentados, com renda e casa próprias. Todos eles possuem condições adequadas de eletricidade e saneamento básico, e $78,6 \%$ possuem telefone fixo. Metade da amostra reside com apenas mais uma pessoa, e 42,6\% possuem animal de estimação.

No que concerne às doenças dos idosos, 78,6\% apresentam problemas cardíacos, 64,3\% metabólicos, $42,9 \%$ neurológicos, $21,4 \%$ osteomusculares e $64,3 \%$ outros problemas, como depressão, catarata e neoplasias. 
Dentre os 14 cuidadores, apenas um relatou ter realizado capacitação específica para a tarefa. Quanto à ocupação, a maioria $(57,1 \%)$ é do lar, seguido de $21,4 \%$ de aposentados. Nove cuidadores $(64,3 \%)$ não possuem renda própria. A presença de problemas de saúde foi referida por $92,9 \%$ dos cuidadores, e as doenças que mais os acometem são cardíacas (50\%) e metabólicas (35,7\%). A necessidade de medicação regular esteve presente em 57,1\% da amostra de cuidadores, e o gasto mensal para esse fim variou de $\mathrm{R} \$ 10$ a $\mathrm{R} \$ 300$, visto que muitos medicamentos são fornecidos gratuitamente pelo Ministério da Saúde.

A respeito da relação entre cuidador e idoso, $64,3 \%$ dos cuidadores são filhos dos idosos, seguido de $28,6 \%$ que são cônjuges e $7,1 \%$ relataram ser irmãos. Quase a totalidade $(85,7 \%)$ reside na mesma casa que o idoso, e o tempo de morada conjunta varia de três meses a 65 anos. Dentre os cuidadores, $64,3 \%$ relataram receber auxílio de mais uma pessoa para cuidar do idoso.

Ao analisarmos o tempo dedicado ao cuidado: $42,8 \%$ desempenham a atividade de um a três anos; $28 \%$ de quatro a seis anos; $7,1 \%$ de sete a nove anos e $21,4 \%$ há dez ou mais anos. O tempo diário destinado à atividade varia de duas a 24 horas, e a maioria $(78,6 \%)$ relata cuidado de 24 horas por dia.

Em relação ao nível de independência do idoso, avaliado através do índice de Katz, 28,6\% foram classificados como independentes, 50\% como tendo dependência moderada e 21,4\% como muito dependentes. Não houve associação entre intensidade de dor do cuidador e o nível de independência do idoso, tampouco entre intensidade de dor e sobrecarga. Podemos observar que são frequentes os relatos de problemas nas diferentes regiões do corpo nos últimos 12 meses e nos últimos sete dias, como apresentado na Tabela 2.

Tabela 2. Distúrbios musculoesqueléticos referidos pelos cuidadores. Porto Alegre - RS, 2016

\begin{tabular}{|c|c|c|c|c|}
\hline & $\begin{array}{c}\text { Problemas } \\
\text { últimos } \\
12 \text { meses }\end{array}$ & $\begin{array}{l}\text { Impedimento } \\
\text { últimos } \\
12 \text { meses }\end{array}$ & $\begin{array}{c}\text { Consulta } \\
\text { últimos } \\
12 \text { meses }\end{array}$ & $\begin{array}{l}\text { Problemas } \\
\text { últimos } 7 \text { dias }\end{array}$ \\
\hline & $N(\%)$ & $N(\%)$ & $N(\%)$ & $N(\%)$ \\
\hline Pescoço & $2(14,2)$ & - & - & $2(14,2)$ \\
\hline Ombros & $7(50)$ & $1(7,1)$ & $1(7,1)$ & $4(28,6)$ \\
\hline Parte superior das costas & $7(50)$ & $3(21,3)$ & $4(28,6)$ & $5(35,7)$ \\
\hline Cotovelos & $4(28,6)$ & $1(7,1)$ & $1(7,1)$ & $1(7,1)$ \\
\hline Punho/Mãos & $6(42,8)$ & $1(7,1)$ & $1(7,1)$ & $2(14,2)$ \\
\hline Parte inferior das costas & $9(64,3)$ & $4(28,6)$ & $4(28,6)$ & $8(57,1)$ \\
\hline Quadril/Coxas & $9(64,3)$ & $3(21,3)$ & $2(14,2)$ & $7(50)$ \\
\hline Joelhos & $9(64,3)$ & $3(21,3)$ & $3(21,3)$ & $7(50)$ \\
\hline Tornozelos/Pés & $8(57,1)$ & $2(14,2)$ & $1(7,1)$ & $5(35,7)$ \\
\hline
\end{tabular}

Houve correlação forte e inversa entre o nível de independência do idoso e a sobrecarga do cuidador $(\rho=-0,619 ; p=0,018)$. Ao avaliarmos a relação da sobrecarga separadamente por seus domínios, houve correlação entre nível de independência do idoso apenas com sobrecarga tempo dependente $(\rho=-0,866 ; p=0,000)$ e sobrecarga à vida pessoal $(\rho=-0,708$; $p=0,005$ ). A Tabela 3 apresenta os dados de dor e de sobrecarga do cuidador em relação ao nível de independência do idoso.

Tabela 3. Ocorrência de dor e sobrecarga do cuidador em relação ao nível de independência do idoso. Porto Alegre - RS, 2016

\begin{tabular}{cccc}
\hline & Dor cuidador (EVA) & Sobrecarga cuidador \\
Independência do idoso (Katz) Muito dependente & Média $\pm \mathbf{d p}$ & & Média $\pm \mathbf{d p}$ \\
\hline Moderado & $5,47 \pm 4,78$ & & $39,3 \pm 9,81$ \\
\hline Independente & $4,67 \pm 2,70$ & $35,0 \pm 8,10$ \\
\hline
\end{tabular}




\section{DISCUSSÃO}

No presente estudo foi avaliada a associação entre sobrecarga e dor em cuidadores principais de idosos com o nível de independência do idoso. Houve predomínio de idosos do sexo feminino (57,1\%) e com baixo nível de escolaridade (primeiro grau incompleto, 64,3\%). A média de idade dos idosos foi de 82,14 anos. Embora não exista influência direta entre a idade do idoso e o grau de sobrecarga do cuidador, devemos ressaltar que, quanto maior a idade do idoso, maior é o risco de morbidades que influenciam seu nível de dependência, podendo acarretar, assim, maior sobrecarga ao cuidador?

Quanto ao perfil de cuidadores, houve prevalência do sexo feminino, e o grau de parentesco com o idoso foi predominantemente de filho(a). Esses achados corroboram diversos estudos publicados na área ${ }^{13,14,20-22}$. Pode-se atribuir tal hegemonia feminina à bagagem histórica $\mathrm{e}$ cultural que atribui à mulher a função de cuidadora do lar e da família ${ }^{13}$. Atualmente, as mulheres estão conquistando mais espaço na sociedade e, muitas vezes, submetendo-se à dupla jornada, o que pode acarretar considerável sobrecarga e contribuir para o comprometimento da sua qualidade de vida. Outro fato importante a ser destacado é a média de idade dos cuidadores (66 anos), demonstrando que são, principalmente, idosos, já autorreferindo diversas doenças, responsáveis pelo cuidado de outros idosos.

Nossos resultados divergem dos encontrados em pesquisa espanhola ${ }^{23} \mathrm{com} 43$ cuidadores principais de idosos dependentes, em que houve relação direta entre intensidade da dor, mensurada pela EVA, e grau de sobrecarga do cuidador. Na pesquisa de Aguilar et al. ${ }^{23}$, os cuidadores eram em sua maioria (95\%) familiares e ainda, como critério de inclusão, apresentavam dor aguda nos últimos sete dias ou dor crônica. Em nosso estudo, 28,6\% dos cuidadores não apresentaram dor na última semana, o que de fato pode representar um viés de comparação com o estudo espanhol. Todos os cuidadores da nossa amostra tinham grau de parentesco com o idoso e não eram remunerados para exercer o cuidado; já na pesquisa espanhola, $5 \%$ não tinham parentesco com o idoso dependente e $23,3 \%$ recebiam remuneração para cuidar. Apesar de compor uma pequena porção da amostra, a familiaridade e a remuneração podem interferir na autopercepção de dor e de sobrecarga. Apesar de não ter havido correlação estatisticamente significativa, verificamos que as médias de intensidade de dor foram crescentes quanto maior dependência do idoso (Tabela 3).

Houve correlação forte e inversa entre o nível de independência do idoso e a sobrecarga do cuidador ( $\rho=-0,619 ; p=0,018)$, ou seja, quanto mais independente o idoso, menor a sobrecarga sofrida pelo cuidador. Wachholz et al. ${ }^{20}$ também verificaram correlação entre o grau de dependência funcional do idoso e o nível de sobrecarga percebida pelo cuidador em estudo envolvendo 45 cuidadores familiares de idosos frágeis.

Ao avaliarmos a relação da sobrecarga separadamente por seus domínios, houve correlação entre nível de independência do idoso apenas com sobrecarga tempo dependente $(\rho=-0,866 ; p=0,000)$ e sobrecarga à vida pessoal $(\rho=-0,708 ; p=0,005)$. Optamos por avaliar a sobrecarga do cuidador através do Caregiver Burden Inventory (Inventário de Sobrecarga do Cuidador), adaptado e validado para o português. Este instrumento oferece uma medida multidimensional da sobrecarga, com escores separados para cada dimensão, ao invés de um escore global ou unidimensional. Ainda que o instrumento mais utilizado para avaliar sobrecarga do cuidador em pesquisas brasileiras ${ }^{5,8,21,24-26}$ seja o Burden Interview, a adoção de uma avaliação multidimensional para sobrecarga do cuidador foi relevante em nosso estudo. Valer et al. ${ }^{15}$ salientam que o escore global tende a mascarar as diferenças entre os níveis de sobrecarga nos diferentes domínios do cuidado e não permite a investigação de perfis de sobrecarga do cuidador.

As demandas de cuidado de um idoso dependente podem desencadear sobrecarga e dor no cuidador ${ }^{24}$. Embora não haja consenso na literatura em relação ao tempo de cuidado e o nível de impacto no cuidador ${ }^{27,28}$, neste estudo foi possível observar uma forte relação entre o nível independência do idoso e a sobrecarga tempo dependente. Aparentemente, quanto mais dependente é o idoso, maior é o cuidado e a atenção despendida pelo cuidador para o auxílio da realização de AVDs. Inclusive, o domínio tempo dependente proposto pelo Inventário de Sobrecarga do Cuidador relaciona-se às necessidades de cuidado para a realização das 
AVDs contempladas no Índice Katz. Ainda, a quantidade de tempo dispensado à atividade de cuidado sobrecarrega os cuidadores e já foi abordada por outros trabalhos ${ }^{29,30}$ que ratificam os resultados aqui encontrados.

Já o domínio de sobrecarga à vida pessoal proposto pelo Inventário de Sobrecarga do Cuidador remete às abdicações impostas pelo cuidado. De fato, cuidadores sentem-se sobrecarregados pelas alterações da vida pessoal exigidas pela sobreposição de tarefas referentes ao ato de cuidar, de modo a renunciarem a suas próprias necessidades ${ }^{6,13}$. No Brasil, e em muitos outros países, a tendência atual é recomendar a permanência dos idosos incapacitados em suas casas sob os cuidados de suas famílias. Ao assumir essa responsabilidade, o cuidador abdica de muitos interesses pessoais e passa a ter restrições em relação à sua própria vida ${ }^{24}$.

Não houve associação entre intensidade de dor e o nível de independência do idoso. Apesar disso, podemos observar que são frequentes os relatos de sintomas musculoesqueléticos na parte inferior das costas, quadril/coxas e joelhos, nos últimos 12 meses e nos últimos sete dias. Em um estudo que avaliou a prevalência de distúrbios musculoesqueléticos em 105 auxiliares e técnicos de enfermagem, cujas funções exercidas são semelhantes às de um cuidador de idoso, os locais mais referidos foram: lombar (59\%), ombros (40\%) e joelhos $(33,3 \%)^{31}$. Alencar e Montrezor ${ }^{32}$, em estudo com trabalhadores de instituições de longa permanência de idosos, também mostraram alta prevalência de sintomas dolorosos osteomusculares nessa população, sendo a região mais acometida a lombar (44\%). A manifestação de distúrbio musculoesquelético lombar pode estar relacionada às atividades de cuidado com os idosos e às suas condições físicas, gerando, consequentemente, sobrecarga musculoesquelética e má postura ${ }^{33}$.

Os cuidadores sentem-se sobrecarregados devido às inúmeras exigências que o ato de cuidar requerem, além de escassa e inadequada informação que têm a respeito da patologia de base dos idosos dependentes ${ }^{13}$. Quando questionados sobre formação na área da saúde, $92,86 \%$ dos participantes responderam negativamente. Uma revisão integrativa publicada na literatura mostra evidências de que os cuidadores apresentam baixo domínio sobre os problemas de saúde dos idosos e os cuidados necessários ${ }^{34}$. Um dos estudos incluído na revisão aponta que tais achados podem comprometer a terapêutica e acarretar sobrecarga percebida, pois os cuidadores não estão preparados para lidar adequadamente com as especificidades do paciente. Isso ratifica a importância de instruir cuidadores sobre saúde e manejo de idosos dependentes e autocuidado.

Quanto às limitações do estudo, pode-se citar o tamanho reduzido da amostra e o fato de os participantes pertencerem a uma única região distrital de Porto Alegre. Assim, estudos nas demais regiões que avaliem o perfil e as relações entre nível de independência dos idosos e a dor e sobrecarga de seus cuidadores principais se fazem necessários para melhor reconhecimento dessa população, fornecendo subsídios para implementação de políticas públicas direcionadas à prevenção de agravos e promoção da saúde em cuidadores.

Este estudo evidenciou forte correlação entre sobrecarga do cuidador e nível de independência dos idosos, mostrando que quanto mais independente for o idoso, menor será a sobrecarga atribuída ao seu cuidador. Quando avaliado separadamente nos diferentes domínios do questionário, houve correlação entre o nível de independência do idoso e sobrecarga tempo dependente e à vida pessoal. Em relação à ocorrência de dor, não se verificou correlação desta variável à sobrecarga do cuidador e ao nível de independência do idoso.

Fatores relacionados à sobrecarga dos cuidadores de idosos dependentes são passíveis de modificação. Portanto, avaliar e monitorar este aspecto, bem como verificar possíveis associações às características funcionais dos idosos, contribui para o desenvolvimento de ações em prevenção de danos e promoção de saúde para essa população.

\section{REFERÊNCIAS}

1. Barros DSL, Silva DLM, Leite SN. Conduta do tratamento medicamentoso por cuidadores de idosos. Interface 2015;19(54):527-36.

2. Brasil. Ministério da Saúde. Portaria n $2.528,19$ de outubro de 2006. Aprova a Política Nacional de Saúde da Pessoa Idosa. Diário Oficial da União, Brasília, 20 de outubro de 2006; Seção 1. p. 149. 
3. Gratão ACM, Talmelli LFS, Figueiredo LC, Rosset I, Freitas CP, Rodrigues RAP. Dependência funcional de idosos e a sobrecarga do cuidador. Rev Esc Enferm USP. 2013;47(1):137-44. http://dx.doi.org/10.1590/ S0080-62342013000100017. PMid:23515813.

4. Fernandes BCW, Ferreira KCP, Marodin MF, Val MON, Fréz AR. Influência das orientações fisioterapêuticas na qualidade de vida e na sobrecarga de cuidadores. Fisioter Mov. 2013 Mar;26(1):151-8. http://dx.doi. org/10.1590/S0103-51502013000100017.

5. Fuhrmann AC, Becker Kottwitz Bierhals CC, Santos NO, Paskulin LM. Associação entre a capacidade funcional de idosos dependentes e a sobrecarga do cuidador familiar. Rev Gaúcha Enferm. 2015 Mar;36(1):14-20. http://dx.doi.org/10.1590/1983-1447.2015.01.49163. PMid:26098797.

6. Vieira ALT, Rodrigues TFC, Gomes Segundo LV, Lima RF, Melo EG. Avaliação do estresse em cuidadores de idosos na unidade de saúde da família "Vila Saúde". Rev M\&P 2015;1(1):61-74.

7. Fernandes MGM, Garcia TR. Atributos da tensão do cuidador familiar de idosos dependentes. Rev Esc Enferm USP. 2009 Dec;43(4):818-24. http://dx.doi.org/10.1590/S0080-62342009000400012. PMid:20085151.

8. Gratão ACM, Vendrúscolo TRP, Talmelli LFS, Figueiredo LC, Santos JLF, Rodrigues RAP. Sobrecarga e desconforto emocional em cuidadores de idosos. Texto Contexto Enferm. 2012 Jun;21(2):304-12. http:// dx.doi.org/10.1590/S0104-07072012000200007.

9. Mota FRN, Victor JF, Silva MJ, Bessa MEP, Amorim VL, Cavalcante MLSN, et al. Adaptação transcultural do Caregiver Reaction Assessment para uso no Brasil com cuidadores informais de idosos. Rev Esc Enferm USP. 2015;49(3):424-31. http://dx.doi.org/10.1590/S0080-623420150000300010. PMid:26107703.

10. Borsje P, Hems MA, Lucassen PL, Bor H, Koopmans RT, Pot AM. Psychological distress in informal caregivers of patients with dementia in primary care: course and determinants. Fam Pract. 2016 Aug;33(4):374-81. http://dx.doi.org/10.1093/fampra/cmw009. PMid:26969508.

11. Garlo K, O'Leary JR, Van Ness PH, Fried TRJ. Burden in caregivers of older adults with advanced illness. J Am Geriatr Soc. 2010 Dec;58(12):2315-22. http://dx.doi.org/10.1111/j.1532-5415.2010.03177.x. PMid:21087225.

12. Bevans M, Sternberg EM. Caregiving burden, stress, and health effects among family caregivers of adult cancer patients. JAMA. 2012 Jan;307(4):398-403. http://dx.doi.org/10.1001/jama.2012.29. PMid:22274687.

13. Costa ECS, Pereira PD, Miranda RAP, Bastos VHV, Machado DCD. Sobrecarga física e mental dos cuidadores de pacientes em atendimento fisioterapêutico domiciliar das Estratégias de Saúde da Família de Diamantina (MG). Rev Baiana de Saúde Pública. 2013 Jan-Mar;37(1):133-50.

14. Loureiro LSN, Fernandes MGM, Nóbrega MML, Rodrigues RAP. Sobrecarga em cuidadores familiares de idosos: associação com características do idoso e demanda de cuidado. Rev Bras Enferm. 2014 MarApr;67(2):227-32. PMid:24861065.

15. Valer DB, Aires M, Fengler FL, Paskulin LMG. Adaptação e validação do Inventário de Sobrecarga do Cuidador para uso em cuidadores de idosos. Rev. Latino- Am. Enfermagem. 2015 Jan-Feb;23(1):130-8. http://dx.doi.org/10.1590/0104-1169.3357.2534.

16. de Barros ENC, Alexandre NMC. Cross-cultural adaptation of the Nordic musculoskeletal questionnaire. Int Nurs Rev. 2003 Jun;50(2):101-8. http://dx.doi.org/10.1046/j.1466-7657.2003.00188.x. PMid:12752909.

17. Pinheiro FA, Troccoli BT, de Carvalho CV. Validação do Questionário Nórdico de Sintomas Osteomusculares como medida de morbidade. Rev Saude Publica. 2002 Jun;36(3):307-12. http://dx.doi.org/10.1590/S003489102002000300008. PMid:12131969.

18. Martinez JE, Grassi DC, Marques LG. Análise da aplicabilidade de três instrumentos de avaliação de dor em distintas unidades de atendimento: ambulatório, enfermaria e urgência. Rev Bras Reumatol. 2011 Aug;51(4):304-8. http://dx.doi.org/10.1590/S0482-50042011000400002.

19. Duarte $Y A O$, Andrade CL, Lebrão ML. O Índex de Katz na avaliação da funcionalidade dos idosos. Rev Esc Enferm USP. 2007 Jun;41(2):317-25. http://dx.doi.org/10.1590/S0080-62342007000200021. PMid:17722401.

20. Wachholz PA, Santos RCC, Wolf LSP. Reconhecendo a sobrecarga e a qualidade de vida de cuidadores familiares de idosos frágeis. Rev Bras Geriatr Gerontol. 2013 Sep;16(3):513-26. http://dx.doi.org/10.1590/ S1809-98232013000300010.

21. Nardi EFR, Sawada NO, Santos JLF. Associação entre a incapacidade funcional do idoso e a sobrecarga do cuidador familiar. Rev Latino-Am Enfermagem. 2013 Oct;21(5):1096-103. http://dx.doi.org/10.1590/ S0104-11692013000500012. 
22. Souza LR, Hanus AJ, Libera LBD, Silva VM, Mangilli EM, Simões PW, et al. Sobrecarga no cuidado, estresse e impacto na qualidade de vida de cuidadores domiciliares assistidos na atenção básica. Cad Saude Colet. 2015;23(2):140-9. http://dx.doi.org/10.1590/1414-462X201500020063.

23. Aguilar LV, Peña MAS, Ponce GC. Sobrecarga y dolor percebido en cuidadoras de ancianos dependientes. Enferm Glob. 2012 jul;11(27):159-64.

24. Silva CF, Passos VMA, Barreto SM. Frequência e repercussão da sobrecarga de cuidadoras familiares de idosos com demência. Rev Bras Geriatr Gerontol. 2012 dec;15(4):707-31. http://dx.doi.org/10.1590/S180998232012000400011.

25. Loureiro LSN, Fernandes MGM, Marques S, Nobrega MML, Rodrigues RAP. Sobrecarga de cuidadores familiares de idosos: prevalência e associação com características do idoso e do cuidador. Rev Esc Enferm USP. 2013 Oct;47(5):1129-36. http://dx.doi.org/10.1590/S0080-623420130000500017.

26. Stackfleth R, Diniz MA, Fhon JRS, Vendruscolo TRP, Fabrício-Whebe SCC, Marques S, et al. Sobrecarga de trabalho em cuidadores de idosos fragilizados que vivem no domicílio. Acta Paul Enferm. 2012;25(5):76874. http://dx.doi.org/10.1590/S0103-21002012000500019.

27. Garrido R, Menezes PR. Impacto em cuidadores de idosos com demência atendidos em um serviço psicogeriátrico. Rev Saude Publica. 2004 Dec;38(6):835-41. http://dx.doi.org/10.1590/S003489102004000600012. PMid:15608902.

28. Cruz MN, Hamdan AC. O impacto da doença de Alzheimer no cuidador. Psicol Estud. 2008 Jun;13(2):223-9. http://dx.doi.org/10.1590/S1413-73722008000200004.

29. Fernandes MGM, Garcia TR. Determinantes da tensão do cuidador familiar de idosos dependentes. Rev Bras Enferm. 2009 Feb;62(1):57-63. http://dx.doi.org/10.1590/S0034-71672009000100009. PMid:19219355.

30. Floriani CA, Schramm FR. Atendimento domiciliar ao idoso: problema ou solução? Cad Saude Publica. 2004;20(4):986-94. http://dx.doi.org/10.1590/S0102-311X2004000400013. PMid:15300291.

31. Gurgueira GP, Alexandre NMC, Corrêa Filho HR. Prevalência de sintomas músculo-esqueléticos em trabalhadoras de enfermagem. Rev. Latino-Am. Enfermagem. 2003 Oct;11(5):608-13. http://dx.doi. org/10.1590/S0104-11692003000500007.

32. Alencar MCB, Montrezor JB. Aspectos da organização do trabalho e os distúrbios osteomusculares: um estudo com trabalhadores em instituições de longa permanência de idosos. Rev Ter Ocup Univ Sao Paulo. 2010 Jan-Apr;21(1):15-22. http://dx.doi.org/10.11606/issn.2238-6149.v21i1p15-22.

33. Karsch UM. Idosos dependentes: famílias e cuidadores. Cad Saude Publica. 2003 Jun;19(3):861-6. http:// dx.doi.org/10.1590/S0102-311X2003000300019. PMid:12806489.

34. Oliveira DC, D'Elboux MJ. Estudos nacionais sobre cuidadores familiares de idosos: revisão integrativa. Rev Bras Enferm. 2012 Oct;65(5):829-38. http://dx.doi.org/10.1590/S0034-71672012000500017. PMid:23338589. 